\title{
openheart Cardiac stereotactic ablative radiotherapy for control of refractory ventricular tachycardia: initial UK multicentre experience
}

\author{
Justin Lee (D) , ${ }^{1}$ Matthew Bates, ${ }^{2}$ Ewen Shepherd, ${ }^{3}$ Stephen Riley, ${ }^{4}$ \\ Michael Henshaw, ${ }^{4}$ Peter Metherall, ${ }^{5}$ Jim Daniel, ${ }^{6}$ Alison Blower, ${ }^{6}$ David Scoones, ${ }^{7}$ \\ Michele Wilkinson, ${ }^{8}$ Neil Richmond, ${ }^{8}$ Clifford Robinson, ${ }^{9}$ Phillip Cuculich, ${ }^{9}$ \\ Geoffrey Hugo, ${ }^{9}$ Neil Seller, ${ }^{3}$ Ruth McStay, ${ }^{10}$ Nicholas Child, ${ }^{2}$ Andrew Thornley, ${ }^{2}$ \\ Nicholas Kelland, ${ }^{1}$ Philip Atherton, ${ }^{8}$ Clive Peedell, ${ }^{6}$ Matthew Hatton ${ }^{4}$
}

\section{- Additional supplemental material is published online only. To view, please visit the journal online (http://dx.doi.org/10. 1136/openhrt-2021-001770) \\ To cite: Lee J, Bates M, Shepherd E, et al. Cardiac stereotactic ablative radiotherapy for control of refractory ventricular tachycardia: initial UK multicentre experience. Open Heart 2021;8:e001770. doi:10.1136/ openhrt-2021-001770}

Received 23 July 2021 Accepted 1 November 2021
Check for updates

(C) Author(s) (or their employer(s)) 2021. Re-use permitted under CC BY-NC. No commercial re-use. See rights and permissions. Published by BMJ.

For numbered affiliations see end of article.

Correspondence to Dr Justin Lee; justin.lee@nhs. net

\section{ABSTRACT}

Background Options for patients with ventricular tachycardia (VT) refractory to antiarrhythmic drugs and/or catheter ablation remain limited. Stereotactic radiotherapy has been described as a novel treatment option.

Methods Seven patients with recurrent refractory VT, deemed high risk for either first time or redo invasive catheter ablation, were treated across three UK centres with non-invasive cardiac stereotactic ablative radiotherapy (SABR). Prior catheter ablation data and noninvasive mapping were combined with cross-sectional imaging to generate radiotherapy plans with aim to deliver a single 25 Gy treatment. Shared planning and treatment guidelines and prospective peer review were used. Results Acute suppression of VT was seen in all seven patients. For five patients with at least 6 months followup, overall reduction in VT burden was $85 \%$. No highgrade radiotherapy treatment-related side effects were documented. Three deaths (two early, one late) occurred due to heart failure.

Conclusions Cardiac SABR showed reasonable VT suppression in a high-risk population where conventional treatment had failed.

\section{INTRODUCTION}

Structural heart disease and impaired ventricular function increase the risk of lifethreatening arrhythmia including ventricular tachycardia (VT). Current approaches to the management of VT involve the placement of implantable cardioverter defibrillators (ICDs), use of antiarrhythmic drugs (AADs) and/or catheter ablation. ${ }^{1}$ Although ICDs improve patient survival, ICD shocks can be detrimental to quality of life and are associated with poor prognosis. ${ }^{2}$ AADs have modest efficacy and can have significant side effects. ${ }^{3}$ Catheter ablation is effective for control of VT and reduction in ICD shocks, but also represents a prolonged, technically challenging

\section{Key questions}

What is already known about this subject?

- Patients with uncontrolled ventricular arrhythmias related to cardiac scar tend to have a poor prognosis and significant morbidity. This is related to both frequent arrythmia episodes and treatment thereof, as well as their underlying cardiac dysfunction.

- Stereotactic radiotherapy (SABR) is an established treatment for many cancers.

What does this study add?

- We report initial UK experience with cardiac SABR treatment of refractory ventricular arrhythmias.

- Across three centres, a multidisciplinary approach with collaboration between cardiology (electrophysiology and imaging) and oncology teams (clinical oncology and medical physics) was employed to successfully deliver treatment.

How might this impact on clinical practice?

- This represents an exciting and novel non-invasive treatment option, but further clinical trials, refinements in technique and mechanistic studies are needed.

procedure that may pose higher risks to more frail patients. ${ }^{45}$

Stereotactic radiotherapy (SABR) is a technique for treating targets anywhere in the body, made possible by advances in radiation treatment planning, imaging guidance and delivery systems. High doses of radiation can be precisely delivered to defined targets, with a steep drop off in dose gradients to minimise dose to surrounding normal tissues. SABR achieves very high local tumour control rates with low toxicity and has been widely adopted in the management of a range of cancers, including lung, prostate, liver, kidney and pancreas. ${ }^{6}$ Following the first patient 
treatment in $2012,{ }^{7}$ several single centre case series ${ }^{8} 9$ and a Phase I/II study (EP-guided Noninvasive Cardiac Radioablation for treatment of Ventricular Tachycardia $(\text { ENCORE-VT) })^{10}$ have demonstrated the potential for non-invasive cardiac SABR treatment to control VT where conventional treatment has failed. However, to date the entire literature describes less than 60 patients treated by this novel approach. ${ }^{11-13}$ Recognising the urgent need for more effective treatments in patients with refractory VT, three UK tertiary cardiac centres agreed to collaborate in developing cardiac SABR services aiming to maximise the benefits of combined experience of a novel technique. We now report our initial experiences.

\section{METHODS}

Each institution obtained formed a local cardiology and oncology working group and obtained institutional approval for use of the established SABR service in a novel compassionate use indication. Working with the ENCORE-VT trial group, ${ }^{10}$ planning, treatment and follow-up guidelines were developed and shared.

Over the period June 2019 to January 2020, seven patients with recurrent VT despite AAD use and prior invasive VT ablation or contra-indication to invasive VT ablation underwent cardiac SABR treatment. All patients received acute initial stabilisation measures including correction of electrolytes, optimisation of heart failure management and additional AADs where appropriate. All patients were discussed in cardiology and oncology meetings at each local centre, and also with the other two UK centres prior to proposing SABR treatment. All patients gave interdisciplinary informed consent and treatment was delivered on outpatient basis with option of overnight hospital admission post-treatment permitted at clinician discretion due to novelty of cardiac SABR treatment. Patient characteristics are summarised in table 1.

\section{Pre-treatment workup}

Four-dimensional (4D) high-resolution cardiac and respiratory cycle (radiotherapy planning) CT scans were both performed with intravenous contrast (and oral contrast if deemed necessary to demonstrate gastrointestinal tract for inferior wall targets) and co-registered. The cardiac CT scan was used to define cardiac structures in particular regions of scar based on myocardial wall thickness, and the radiotherapy planning CT scan was used to define extracardiac structures. Cardiac MRI and nuclear medicine scans were also used to define myocardial scar where available.

Clinical 12 lead ECGs of VT and prior invasive electrophysiology data, including electroanatomic voltage and activation maps, were reviewed by two electrophysiologists from each centre to agree on VT exit sites. In six of seven patients, inducibility of clinical VT and other VTs as tolerated was confirmed by a non-invasive electrophysiology study (programmed stimulation through ICD) combined with body surface mapping using the View into Ventricular Onset (VIVO system - Catheter Precision).

In general, the integration of structural and ECG data was performed manually by side to side comparison and clinician consensus. Use of the 17 segment cardiac model was encouraged to facilitate communication between physicians and centres. In Sheffield, an additional step was developed to fuse imaging data (DICOM format) and electroanatomic map (EAM)/VIVO data (.dif or .vtk) using Mimics Innovation Suite, V.23 (Materialise, Leuven, Belgium). Deformable registration techniques were used to fuse the various modalities using the $\mathrm{CT}$ as the primary fixed image for planning review. This permitted a threedimensional target to be defined directly on the myocardial surface and a target volume generated by extruding the surface through the myocardium. This volume was exported to directly generate a DICOM RT structure in Eclipse ( $n=2$, Varian Medical Systems). For the two other

\begin{tabular}{|c|c|c|c|c|c|c|c|c|}
\hline Patient & Age & Gender & LVEF & Aetiology & NYHA & Device & Antiarrhythmic drugs & Prior catheter ablation \\
\hline 1 (STH) & $70 \mathrm{~s}$ & $M$ & 20 & Myocarditis & III & CRTD & $\begin{array}{l}\text { A } 300 \mathrm{mg} \text { once a day } \\
\text { R } 500 \mathrm{mg} \text { two times per day } \\
\text { (intolerant Me) }\end{array}$ & 2 \\
\hline 2 (NUTH) & $70 \mathrm{~s}$ & $\mathrm{~F}$ & 30 & Idiopathic & IV & CRTD & A 200 mg once a day & 3 \\
\hline 3 (JCUH) & $70 \mathrm{~s}$ & $M$ & 45 & Ischaemic & $\|$ & ICD & A 200 mg once a day & 1 \\
\hline 4 (NUTH) & $60 \mathrm{~s}$ & M & 35 & Ischaemic & ॥ & ICD & A $200 \mathrm{mg}$ once a day & 3 \\
\hline 5 (STH) & $60 \mathrm{~s}$ & M & 15 & Ischaemic & $\|$ & CRTD & $\begin{array}{l}\text { Me } 300 \mathrm{mg} \text { three times a day } \\
\text { (prior use of } A, R \text { ) }\end{array}$ & 2 \\
\hline 6 (NUTH) & $70 \mathrm{~s}$ & $\mathrm{~F}$ & 25 & Ischaemic & III & ICD & $\begin{array}{l}\text { A } 200 \mathrm{mg} \text { once a day } \\
\text { (prior use of Me, } \mathrm{P} \text { ) }\end{array}$ & 2 \\
\hline 7 (JCUH) & $70 \mathrm{~s}$ & $\mathrm{~F}$ & 20 & Ischaemic & III & CRTD & A $200 \mathrm{mg}$ once a day & 0 \\
\hline
\end{tabular}

A, amiodarone; CRTD, biventricular implantable defibrillator; F, female; ICD, implantable defibrillator; JCUH, James Cook University Hospital Middlesbrough; LVEF, left ventricular ejection fraction; M, male; Me, mexiletine; NUTH, Newcastle Hospitals NHS Foundation Trust; NYHA, New York Heart Association class; P, propafenone; R, ranolazine; STH, Sheffield Teaching Hospitals; VIVO, view into ventricular onset (noninvasive mapping). 


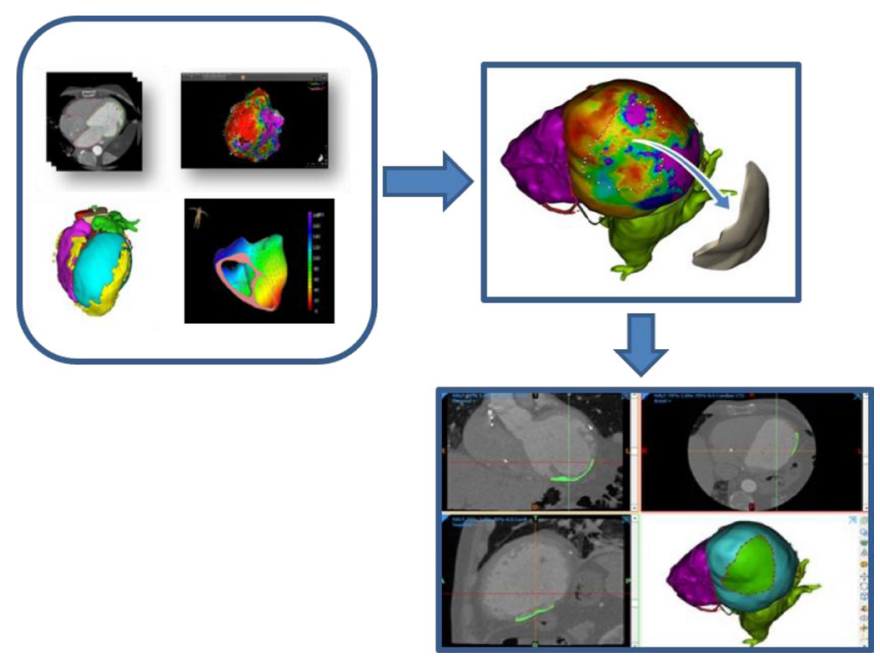

Figure 1 Illustrative workflow-cardiac CT imaging is combined with electrophysiology mapping data into a single three-dimensional model used to define a target region for import into radiotherapy planning software.

centres, segments of scar adjacent to VT exit site location(s) were manually delineated for gross target volume (GTV) on the respiratory gated 4D radiotherapy planning scan using either Monaco ( $n=2$, Middlesbrough, Elekta) or Raystation ( $n=3$, Newcastle, RaySearch Laboratories) software. Illustrative workflow for the image fusion approach to define the cardiac target is presented in figure 1 and a summary of target selection data is given in table 2.

Cardiac GTV margins were expanded to account for cardiac and respiratory motion to generate the internal target volume. Finally, a $3-5 \mathrm{~mm}$ margin was added to account for variation in patient positioning thus generating the final planning target volume (PTV). Treatment plans were produced with aim to achieve single dose 25 Gy treatment to $95 \%$ of PTV-see example in figure 2. Dose constraints to off target structures such as thoracic and abdominal organs were based on the ENCORE-VT protocol and American Association of Physicists in Medicine guidelines. ${ }^{1415}$ Dose was also minimised to device

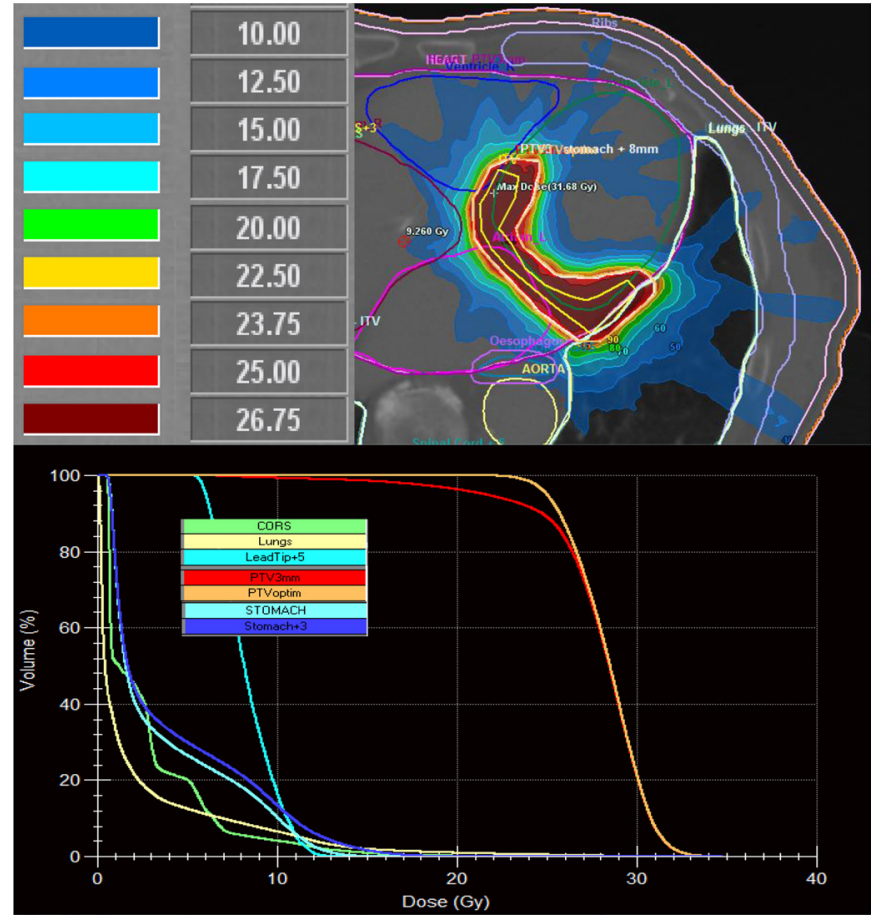

Figure 2 Example cardiac SABR plan from patient 3-top panel 'isodose' areas show how 25 Gy SABR treatment is focused on cardiac target area, bottom panel-dose-volume histogram demonstrating how treatment to off-target organs at risk is minimised. CORS, coronary arteries; PTVoptim, optimised and prescribed planning target volume; SABR, stereotactic radiotherapy.

lead tips and proximal coronary arteries. In two patients with inferior wall targets, the stomach was in close proximity and at risk of exceeding pre-specified $17.4 \mathrm{~Gy}$ organ at risk limit. Two different approaches were used-in patient 1, the overall SABR dose was reduced to $20 \mathrm{~Gy}$, while in patient 3 , stomach plus $8 \mathrm{~mm}$ margin was removed from the PTV. Further detailed information on SABR plans is provided in the online supplemental data table 1 as per International Commission on Radiation Units and Measurements (ICRU) 91 reporting standards. ${ }^{16}$

Table 2 Targeting details

\begin{tabular}{|c|c|c|c|c|c|}
\hline Patient & Scar segments (MRI/CT) & $\begin{array}{l}\text { Clinical VT } \\
\text { (12 lead ECG) }\end{array}$ & VIVO NIPS -VT exit & Segments targeted & Treatment \\
\hline 1 (STH) & $10,11,15,16$ & 10,15 & Not performed & 10,15 & Jun 2019 \\
\hline 3 (JCUH) & $3,4,9,10$ & 10 & 3,4 & $3,4,10$ & Jul 2019 \\
\hline 4 (NUTH) & $4,10,15$ & 4 & 10 & 4,10 & Dec 2019 \\
\hline 7 (JCUH) & $2,3,4,5,8,9,10,11,14$ & $N / A^{*}$ & 14 & $2,3,8,9,14$ & Jan 2020 \\
\hline
\end{tabular}

*Device electrogram data available only.

EAM, electroanatomic map; JCUH, James Cook University Hospital Middlesbrough; NIPS, non invasive programmed stimulation; NUTH, Newcastle Hospitals NHS Foundation Trust; STH, Sheffield Teaching Hospitals; VIVO, view into ventricular onset (non-invasive mapping); VT, ventricular tachycardia. 


\begin{tabular}{llcclc}
\hline Table 3 & SABR treatment details & & \\
\hline Patient & VTs targeted & Planning target volume $(\mathbf{m L})$ & Beam on time $(\mathbf{m i n})$ & Linear accelerator & Total time in room $(\mathbf{m i n})$ \\
\hline 1 (STH) & 1 & 65.8 & 7 & Varian & 60 \\
2 (NUTH) & 3 & 57.5 & 5 & Varian & 28 \\
3 (JCUH) & 2 & 139.0 & 12 & Elekta & 40 \\
4 (NUTH) & 1 & 121.1 & 5 & Varian & 35 \\
5 (STH) & 2 & 89.5 & 12 & Varian & 45 \\
6 (NUTH) & 2 & 101.5 & 5 & Varian & 33 \\
7 (JCUH) & 1 & 87.4 & 8 & Elekta & 30 \\
\hline
\end{tabular}

JCUH, James Cook University Hospital Middlesbrough; NUTH, Newcastle Hospitals NHS Foundation Trust; SABR, stereotactic radiotherapy; STH, Sheffield Teaching Hospitals; VT, ventricular tachycardia.

A remote peer-review process was set up between three UK centres and with Center for Non-invasive Cardiac Radioablation, Washington University, St Louis. Anonymised clinical data and treatment plans were uploaded to a secure server and online meetings were held to review case data.

\section{Treatment delivery and follow-up}

During treatment with the Linear Accelerator (Varian Medical Systems/Elekta), departmental patient immobilisation techniques were used which included use of abdominal compression in cases where it was observed to help limit respiratory movement. A Day 0 'practice run' was recommended to give experience with individual patient positioning and cone beam CT registration. Matching was performed on bony structures, device leads and left ventricle outline. Treatment used intensitymodulated planning and delivery. Single fraction highdose treatment was delivered during free breathing without any cardiac fiducial marker gating. See table 3 for summary of treatment delivery. Overnight hospital admission was left at clinician discretion. Most patients had an indication for oral anticoagulation (usually atrial fibrillation), but if not anticoagulation for 4 weeks post SABR was recommended. Patients were then followed with a combination of telephone and in person appointments at 6 weeks and then every 3 months, and through the remote monitoring function of their ICDs. Transthoracic echo was undertaken at 6 weeks, no routine follow-up CT imaging was undertaken. Following the first 6 weeks after SABR, clinicans were encouraged to reduce antiarrhythmics if possible. Patient ICD settings including monitor zones were programmed at the discretion of the local centre, with the common aim of maximising VT detection. During follow-up, all device detected VT episodes were reviewed by an electrophysiologist to confirm the diagnosis.

\section{RESULTS}

\section{Acute observations-first $\mathbf{6 0}$ days}

All patients were successfully treated-see table 3 for specific details of target volume and treatment time. In patient 2, acute termination and suppression of sustained
VT was observed during SABR treatment. Troponin measurement taken 24 hours post SABR in two patients remained in normal range in patient 1 and showed an insignificant rise to less than two times upper limit normal in patient 5. Observed radiotherapy toxicity was limited to Common Terminology Criteria for Adverse Events (CTCAE) Grade 1 fatigue in two patients and no toxicity in terms of lung, cardiac, GI side-effects was observed clinically. No change in LV function was seen on echo at 6 weeks. Patient 1 had an acute flare up of VT post SABR that required temporary escalation of amiodarone and ranolazine doses for 2 months. Patient 3 also experienced further VT post SABR with a different inferior exit site that required escalation of $\mathrm{AADs}$ and then underwent repeat catheter ablation 7 weeks after SABR. No significant changes were noted in the bipolar endocardial voltage map to reflect recent SABR treatment.

Despite acute reduction of VT episodes, patient 2 and patient 7 both died of progressive heart failure within 4 weeks of treatment. Patient 2 was challenging to treat due to lack of anatomic scar on CT/MRI and multiple VT exit sites documented clinically and at VIVO non invasive programmed stimulation (NIPS) involving anterior, posterolateral, summit and apex regions of LV. Both of these patients were either New York Heart Association (NYHA) class IV status at the time of treatment or deteriorated to class IV shortly afterwards. Postmortem histology was obtained in patient 7 with kind permission from patient's family. No acute radiotherapy changes were detected in surrounding organs, though acute changes in the heart were observed-see figure 3. Myocardium in the vicinity of the treated area showed established fibrosis from prior ischaemic damage. Non-fibrotic myocardium showed an increase in capillary vascularity as might be expected in relation to radiation treatment. No other histologic features of radiation exposure such as acute necrosis, changes in blood vessel wall, thrombosis, fibroblast proliferation or nuclear atypia were seen.

\section{VT burden and survival}

Of the remaining five patients, patient 1 died 9 months after treatment due to progressive heart failure, while the others remain alive. Comparing the 6 months prior 


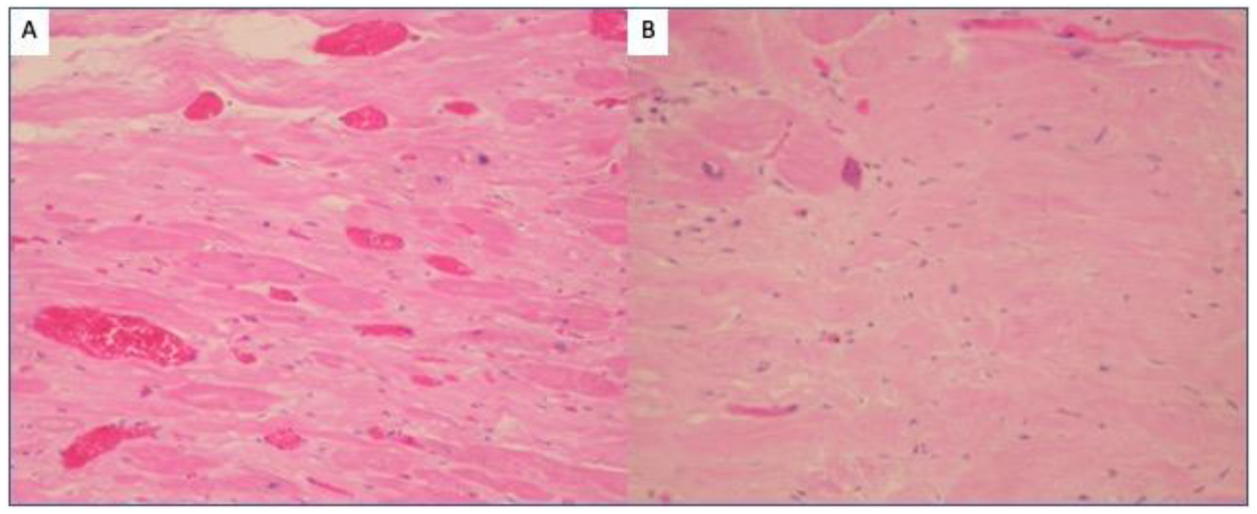

Figure 3 Panel A shows myocardium with some increase in capillary vascularity related to treatment, but minimal fibrosis, within tissue sampled from the targeted area. Panel B shows myocardial fibrosis close to but outside of the targeted area, related to pre-existing myocardial ischaemia.

to treatment with 6 months after treatment we observed $85 \%$ reduction in VT episodes-see figure 4, though in patient 4, the VT detection zones were suboptimally programmed prior to SABR treatment, and the number of episodes of VT prior to SABR was almost certainly undercounted. The majority of VT episodes were either non-sustained or terminated with antitachycardia pacing (ATP). Over the same time period, total ICD shocks across the whole group were reduced from seven pretreatment to none post-treatment.

Following SABR, amiodarone dose was successfully reduced to $100 \mathrm{mg}(\mathrm{n}=2)$ or stopped $(\mathrm{n}=3)$, with the aim of reducing long-term toxicity and improving quality of life.

\section{DISCUSSION}

Our study shows effective (85\%) VT suppression by cardiac SABR, applied on compassionate use basis, in a group of patients with heart failure and recurrent VT and few therapeutic options. Over the past decades, advances in medical therapy and cardiac resynchronisation have yielded improvements in heart failure survival with reduced sudden death. ${ }^{1718}$ Successful VT management is also associated with improved survival, ${ }^{19}$ and reduction in VT episodes improves quality of life by less frequent ICD therapy, fewer hospital admissions and a reduction in AAD use. ${ }^{20}$ However, we accept that we did not formally measure quality of life in this series, and follow-up is limited to 6 months. We have chosen to report total VT episodes as the primary outcome to present the full 'biologic' treatment effect; although ICD shock therapy may be considered a more clinically relevant endpoint, the total numbers of ICD shocks were small in this series.

Given the limited efficacy of second line AADs beyond amiodarone and significant challenges of catheter ablation in frail patients, ${ }^{41}$ non-invasive SABR delivered on an outpatient basis could play an important role in VT management and symptom improvement. The extent of VT suppression seen in our cohort is in keeping with other series. Robinson et al reported $\sim 90 \%$ suppression of VT in 19 patients, ${ }^{10}$ Neuwirth et al $87 \%$ in 10 patients out to median 28 months follow-up. ${ }^{9}$ Gianni et al on the other hand reported good initial effects of treatment in five patients followed for a median 12 months, but significant late recurrences of VT requiring re-escalation of AADs and repeat ablation in three patients. ${ }^{22}$ Lloyd et al reported 10 patients with more modest overall efficacy in terms of VT burden (69\% reduction), ATP (48\% reduction) and ICD shocks (68\% reduction) in an advanced heart failure population. ${ }^{23}$

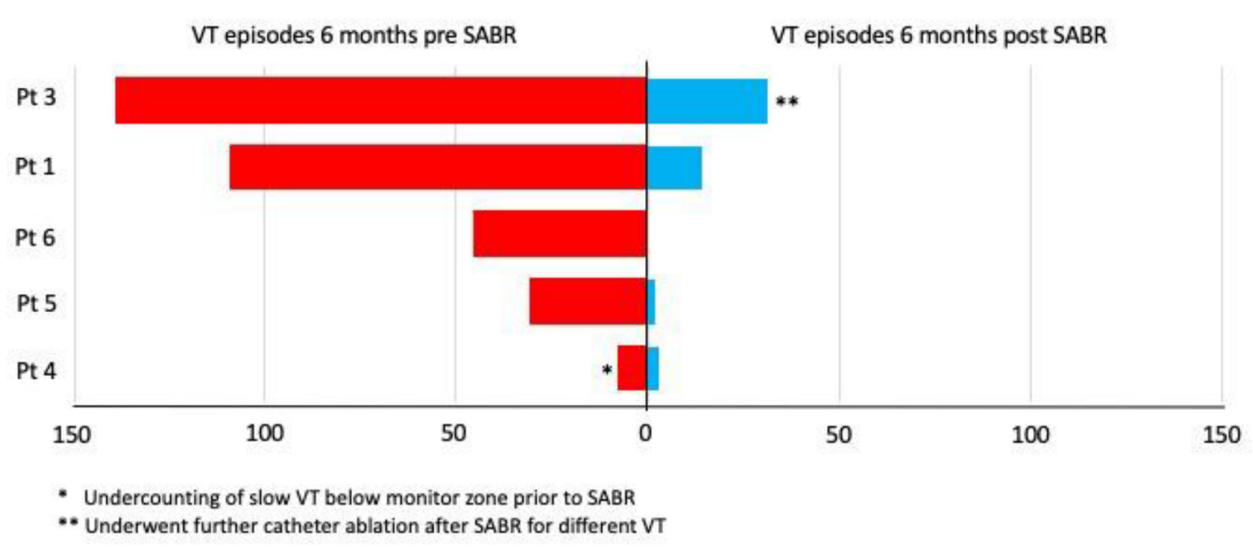

Figure 4 VT episodes in five patients that had at least 6 months follow-up comparing 6 months prior to SABR treatment (red) with 6 months post (blue). SABR, stereotactic radiotherapy; VT, ventricular tachycardia. 
We emphasise that in this series SABR treatment was a 'last resort' procedure for high-risk patients. Unsurprisingly, we observed two acute and one mid-term deaths from non-arrhythmic causes in a cohort with advanced heart failure ${ }^{24}$-all three patients that died were NYHA class III or IV at SABR treatment. The Washington University group has recently reported that long-term survival of their 19 SABR-treated patients may be predicted by baseline cardiac function. ${ }^{25}$ Survivors ( $\mathrm{n}=8$, survival for median of 27.7 months) versus those who received cardiac transplant or died $(\mathrm{n}=11)$ had smaller cardiac volumes on echo and less myocardial scar on MRI. As SABR treatment places less demand on the patient, it is an attractive option for frail patients or those with more advanced heart failure, yet such patients may to be too sick to gain prognostic benefit. Nevertheless, given the ability of SABR to treat substrates inaccessible to catheter ablation, it remains an attractive option for further investigation.

Cardiac SABR target selection is a challenge requiring integration and registration of imaging and functional data. In this series, and reflecting current worldwide experience and literature, we used a range of approaches from manual delineation methods to more sophisticated computer-assisted visualisation methods still under development. ${ }^{26-28}$ The optimum dose and delivery platform for cardiac SABR is yet to be established. We used a single fraction 25 Gy regime as reported by all groups previously. While this did largely suppress VT (in one case at point of delivery), we also saw some short-term VT recurrences which may reflect incomplete substrate ablation. Like Robinson $e t$ al we employed gantry-based linear accelerator systems, while Neuwirth et al and Gianni et al used the Cyberknife platform delivering radiotherapy via a robotic arm. ${ }^{102}$ Cyberknife treatment times are reported as longer-median $80 \mathrm{~min}$ versus $15 \mathrm{~min}$, which could make consistent patient positioning and accurate delivery more challenging, although lower treatment volumes have been reported which might reduce off target effects.

At present, the mechanism(s) of action of cardiac SABR are not established. Alongside other reports, we observed acute VT suppression that seems too rapid to be explained by the development of radiation fibrosis. Our early postmortem data within weeks of SABR demonstrated acute vascular changes but have not shown significant tissue injury or acute fibroblast proliferation, and this is consistent with the postmortem findings of little or no necrosis described by Cuculich et al and also Krug et $a l$ at similar time frames-3 weeks and 57 days post SABR, respectively. ${ }^{89}$ More recently, a four patient series ranging from 12 to 250 days post SABR treatment has reported both cardiac histology and electron microscopy findings of cellular necrosis and fibrosis. ${ }^{30}$ Our observation that troponin levels do not appear to rise acutely, although measured in only two patients, also makes early necrosis as observed in radiofrequency ablation unlikely. Preclinical studies do offer more scope to study radiobiologic effects. Studies in porcine and canine atrial models have confirmed scar formation at 4-6 months with high dose (>32 Gy) treatment. ${ }^{31}{ }^{32}$ Rabbit and dog models have reported a paradoxical acute increase in cardiac conduction velocity following heavy ion irradiation, mediated through increased Connexin 43 expression. ${ }^{33}$ Data taken at serial timepoints up to 4 weeks post irradiation in a rat model did not show necrosis or apoptosis, rather interstitial and intracellular oedema, reflected in slowed conduction with prolongation of PR and QT intervals. ${ }^{34}$ However, any perturbation of myocardial conduction whether increase or decrease may be sufficient to prevent re-entrant VT and most recent data again points to a 'supraphysiologic' phenotype induced by radiation. ${ }^{35}$

The acute and late side effects of radiotherapy also need consideration, including those affecting the heart itself. We did not perform follow-up imaging apart from echo and did not document any clinically significant acute side effects, but long-term effects including pericardial fibrosis and constriction, or coronary and valve disease are well-documented in cancer survivors and are a concern. ${ }^{36}$ There is increasing recognition that even at 12-24 months, survival may be adversely affected in nonsmall cell lung cancer patients receiving higher dose off target treatment to the heart. ${ }^{37}$ Infrequent late effects of cardiac SABR treatment including pericarditis and gastric fistula have been reported as the follow-up of patients treated by this novel technique increases. ${ }^{38}$ Therefore, it is important to report all experience of this treatment, to help refine cardiac SABR techniques.

The unique features of this report are the collaborative approach to application of cardiac SABR across several centres within a larger health network (National Health Service), and the use of different planning and treatment delivery platforms. This report also presents the first use of a remote peer review system for cardiac SABR. SABR is a well-established oncology clinical service and the infrastructure is present in almost all NHS radiotherapy centres in the UK. There is also an established national multidisciplinary group (UK SABR Consortium) that provides expert consensus, quality assurance and liaison with clinical commissioners. The approach to cardiac SABR used so far is a direct translation from standard radiation oncology practice but further refinements will be possible, including improved or semiautomated threedimensional target delineation, ECG or respiratory-gated treatment delivery, and optimisation of dosing and fractionation regimes.

\section{CONCLUSIONS}

Our report confirms, in a UK multicentre setting, prior observations about the short-term and medium-term efficacy and safety of cardiac SABR for control of VT in a high-risk population that was refractory to standard approaches. There is still much to learn about this promising, novel non-invasive treatment and further research is already underway including clinical trials and mechanistic studies. 
Author affiliations

${ }^{1}$ Department of Cardiology, Sheffield Teaching Hospitals NHS Foundation Trust, Sheffield, UK

${ }^{2}$ Department of Cardiology, South Tees Hospital NHS Foundation Trust, Middlesbrough, UK

${ }^{3}$ Department of Cardiology, Newcastle Hospitals NHS Foundation Trust, Newcastle upon Tyne, UK

${ }^{4}$ Weston Park Cancer Centre, Sheffield Teaching Hospitals NHS Foundation Trust, Sheffield, UK

${ }^{5} 3 \mathrm{D}$ Lab, Sheffield Teaching Hospitals NHS Foundation Trust, Sheffield, UK

${ }^{6}$ Department of Oncology, South Tees Hospitals NHS Foundation Trust,

Middlesbrough, UK

${ }^{7}$ Department of Pathology, South Tees Hospital NHS Foundation Trust,

Middlesbrough, UK

${ }^{8}$ Northern Centre for Cancer Care, Newcastle Hospitals NHS Foundation Trust, Newcastle upon Tyne, UK

${ }^{9}$ Center for Noninvasive Cardiac Radioablation, Washington University School of Medicine in St Louis, St Louis, Missouri, USA

${ }^{10}$ Department of Radiology, Newcastle NHS Hospitals Foundation Trust, Newcastle Upon Tyne, UK

\section{Twitter Clive Peedell @cpeedell}

Acknowledgements We also gratefully acknowledge the contributions of $D$ Greenhalgh, K Pilling, R Brooks (Newcastle Hospitals NHS Foundation Trust), M Burgess, J Ainsworth, C Huntley (South Tees NHS Foundation Trust), M Walsh, D Gill, L Siddall (Sheffield Teaching Hospitals NHS Foundation Trust).

Contributors All authors contributed to the conception of the work, or acquisition and interpretation of data. All authors have either written or critically reviewed the paper and given approval for publication. JL is the guarantor of the paper.

Funding The authors have not declared a specific grant for this research from any funding agency in the public, commercial or not-for-profit sectors.

Competing interests None declared.

Patient consent for publication Not applicable.

Ethics approval Institutional approval was given in each centre for compassionate use of SABR for treatment of VT.

Provenance and peer review Not commissioned; externally peer reviewed.

Data availability statement Data are available upon reasonable request.

Open access This is an open access article distributed in accordance with the Creative Commons Attribution Non Commercial (CC BY-NC 4.0) license, which permits others to distribute, remix, adapt, build upon this work non-commercially, and license their derivative works on different terms, provided the original work is properly cited, appropriate credit is given, any changes made indicated, and the use is non-commercial. See: http://creativecommons.org/licenses/by-nc/4.0/.

ORCID iD

Justin Lee http://orcid.org/0000-0003-2444-0169

\section{REFERENCES}

1 Stevenson WG, Wilber DJ, Natale A, et al. Irrigated radiofrequency catheter ablation guided by electroanatomic mapping for recurrent ventricular tachycardia after myocardial infarction: the multicenter thermocool ventricular tachycardia ablation trial. Circulation 2008;118:2773-82.

2 Poole JE, Johnson GW, Hellkamp AS, et al. Prognostic importance of defibrillator shocks in patients with heart failure. $N$ Engl J Med 2008;359:1009-17.

3 Connolly SJ, Dorian P, Roberts RS, et al. Comparison of betablockers, amiodarone plus beta-blockers, or sotalol for prevention of shocks from implantable cardioverter defibrillators: the optic study: a randomized trial. JAMA 2006;295:165-71.

4 Santangeli P, Frankel DS, Tung R, et al. Early mortality after catheter ablation of ventricular tachycardia in patients with structural heart disease. J Am Coll Cardiol 2017;69:2105-15.

5 Frontera A, Panniker S, Breitenstein A, et al. Safety and midterm outcome of catheter ablation of ventricular tachycardia in octogenarians. Europace 2017;19:1369-77.

6 Ball D, Mai GT, Vinod S, et al. Stereotactic ablative radiotherapy versus standard radiotherapy in stage 1 non-small-cell lung cancer
(TROG 09.02 CHISEL): a phase 3, open-label, randomised controlled trial. Lancet Oncol 2019;20:494-503.

7 Loo BW, Soltys SG, Wang L, et al. Stereotactic ablative radiotherapy for the treatment of refractory cardiac ventricular arrhythmia. Circ Arrhythm Electrophysiol 2015;8:748-50.

8 Cuculich PS, Schill MR, Kashani R, et al. Noninvasive cardiac radiation for ablation of ventricular tachycardia. $N$ Engl $J$ Med 2017;377:2325-36

9 Neuwirth R, Cvek J, Knybel L, et al. Stereotactic radiosurgery for ablation of ventricular tachycardia. Europace 2019;21:1088-95.

10 Robinson CG, Samson PP, Moore KMS, et al. Phase I/II trial of electrophysiology-guided noninvasive cardiac Radioablation for ventricular tachycardia. Circulation 2019;139:313-21.

11 van der Ree $\mathrm{MH}$, Blanck O, Limpens J, et al. Cardiac radioablation-a systematic review. Heart Rhythm 2020;17:1381-1392.

12 Kovacs B, Mayinger M, Schindler M, et al. Stereotactic radioablation of ventricular arrhythmias in patients with structural heart disease - a systematic review. Radiother Oncol 2021;162:132-9.

13 Miszczyk M, Jadczyk T, Gołba K, et al. Clinical evidence behind stereotactic radiotherapy for the treatment of ventricular tachycardia (STAR)-a comprehensive Review. J Clin Med 2021;10. doi:10.3390/ jcm10061238. [Epub ahead of print: 1703 2021].

14 Knutson NC, Samson PP, Hugo GD, et al. Radiation therapy workflow and dosimetric analysis from a phase $1 / 2$ trial of noninvasive cardiac radioablation for ventricular tachycardia. Int $J$ Radiat Oncol Biol Phys 2019;104:1114-23.

15 Benedict SH, Yenice KM, Followill D, et al. Stereotactic body radiation therapy: the report of AAPM task group 101. Med Phys 2010;37:4078-101.

16 Wilke L, Andratschke N, Blanck O, et al. ICRU report 91 on prescribing, recording, and reporting of stereotactic treatments with small photon beams : Statement from the DEGRO/DGMP working group stereotactic radiotherapy and radiosurgery. Strahlenther Onkol 2019;195:193-8.

17 Stevenson LW, Pande R, Lynne WS. Witness to progress. Circ Heart Fail 2011:4:390-2.

18 Cleland JGF, Daubert J-C, Erdmann E, et al. The effect of cardiac resynchronization on morbidity and mortality in heart failure. $N$ Engl J Med 2005;352:1539-49.

19 Tung R, Vaseghi M, Frankel DS, et al. Freedom from recurrent ventricular tachycardia after catheter ablation is associated with improved survival in patients with structural heart disease: an international VT Ablation Center Collaborative Group study. Heart Rhythm 2015;12:1997-2007.

20 Martinez BK, Baker WL, Konopka A, et al. Systematic review and meta-analysis of catheter ablation of ventricular tachycardia in ischemic heart disease. Heart Rhythm 2020;17:e206-19.

21 Deyell MW, Steinberg C, Doucette S, et al. Mexiletine or catheter ablation after amiodarone failure in the vanish trial. $J$ Cardiovasc Electrophysiol 2018;29:603-8.

22 Gianni C, Rivera D, Burkhardt JD, et al. Stereotactic arrhythmia radioablation for refractory scar-related ventricular tachycardia. Heart Rhythm 2020;17:1241-8.

23 Lloyd MS, Wight J, Schneider F, et al. Clinical experience of stereotactic body radiation for refractory ventricular tachycardia in advanced heart failure patients. Heart Rhythm 2020;17:415-22.

24 Pocock SJ, Wang D, Pfeffer MA, et al. Predictors of mortality and morbidity in patients with chronic heart failure. Eur Heart $J$ 2006;27:65-75.

25 Zhang S, Navara R, Moore K, et al. Cardiac imaging identifies features associated with reduced survival after noninvasive cardiac radioablation, 2020p.. Available: https://cslide-us.ctimeetingtech. com/hrs20/attendee/eposter/file/1517\#1

26 Boda-Heggemann J, Blanck O, Mehrhof F, et al. Interdisciplinary clinical target volume generation for cardiac Radioablation: multicenter benchmarking for the radiosurgery for ventricular tachycardia (raventa) trial. Int J Radiat Oncol Biol Phys 2021;110:745-56.

27 Hohmann S, Henkenberens C, Zormpas C, et al. A novel opensource software-based high-precision workflow for target definition in cardiac radioablation. $J$ Cardiovasc Electrophysiol 2020;31:2689-95.

28 Brett CL, Cook JA, Aboud AA, et al. Novel workflow for conversion of catheter-based electroanatomic mapping to DICOM imaging for noninvasive Radioablation of ventricular tachycardia. Pract Radiat Oncol 2021;11:84-8.

29 Krug D, Blanck O, Demming T, et al. Stereotactic body radiotherapy for ventricular tachycardia (cardiac radiosurgery) : first-in-patient treatment in Germany. Strahlenther Onkol 2020;196:23-30.

30 Kiani S, Kutob L, Schneider F, et al. Histopathologic and ultrastructural findings in human myocardium after stereotactic 
body radiation therapy for recalcitrant ventricular tachycardia. Circ Arrhythm Electrophysiol 2020;13:e008753.

31 Blanck O, Bode F, Gebhard M, et al. Dose-escalation study for cardiac radiosurgery in a porcine model. Int J Radiat Oncol Biol Phys 2014;89:590-8.

32 Chang JH, Cha M-J, Seo J-W, et al. Feasibility study on stereotactic radiotherapy for total pulmonary vein isolation in a canine model. Sci Rep 2021;11:12369.

33 Amino M, Yoshioka K, Tanabe T, et al. Heavy ion radiation upregulates $\mathrm{C} \times 43$ and ameliorates arrhythmogenic substrates in hearts after myocardial infarction. Cardiovasc Res 2006;72:412-21.

34 Cha M-J, Seo J-W, Kim HJ, et al. Early changes in rat heart after high-dose irradiation: implications for antiarrhythmic effects of cardiac Radioablation. J Am Heart Assoc 2021;10:e019072.
35 Zhang DM, Navara R, Yin T, et al. Cardiac radiotherapy induces electrical conduction reprogramming in the absence of transmural fibrosis. Nat Commun 2021;12:5558.

36 Marmagkiolis K, Finch W, Tsitlakidou D, et al. Radiation toxicity to the cardiovascular system. Curr Oncol Rep 2016;18:15.

37 Speirs CK, DeWees TA, Rehman S, et al. Heart dose is an independent dosimetric predictor of overall survival in locally advanced non-small cell lung cancer. J Thorac Oncol 2017;12:293-301.

38 Robinson CG, Samson P, Moore KMS, et al. Longer term results from a phase I/II study of EP-guided noninvasive cardiac Radioablation for treatment of ventricular tachycardia (ENCORE-VT). Int J Radiat Oncol Biol Phys 2019;105:682. 